\title{
Ranking der dualen Bronchodilatation steigt
}

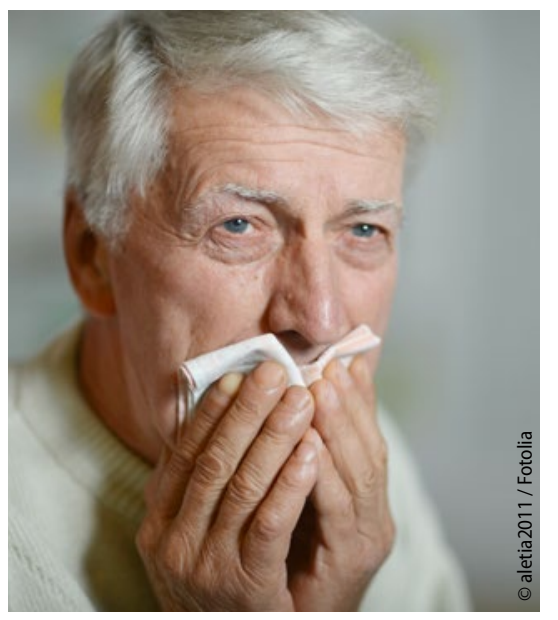

Durch eine duale Bronchodilatation können Auswurf, Husten und Atemnot gelindert werden.

Die 2017 aktualisierten internationalen GOLD-Leitlinien zum Vorgehen bei COPD haben die Rolle der dualen Bronchodilatation mit LAMA plus LABA insgesamt aufgewertet: Bei schwerer Symptomlast kann sie bereits in der Patientengruppe $\mathrm{B}$ eingesetzt werden. In Gruppe $\mathrm{C}$ ist die LABA/LAMA-Kombination erste Wahl als Eskalationsstufe, wenn ein LAMA allein nicht reicht. In Gruppe D wird sie für den Therapiestart empfohlen, mit Eskalation im weiteren Verlauf, falls notwendig. Dies berichtete der Leiter des wissenschaftlichen Komitees der Global Initiative for Chronic Obstructive Lung Disease (GOLD), Prof. Claus Vogelmeier, Universitätsklinikum Gießen/Marburg.

Um einen Patienten einer Gruppe nach GOLD zuzuordnen, müssen künftig nur noch Exazerbationsfrequenz und
Symptomatik erfasst werden. Für letztere empfiehlt Vogelmeier insbesondere den COPD Assessment Test (CAT). Patienten der Gruppe A und B haben 0-1 Exazerbationen ohne stationäre Behandlung; Gruppe A hat wenige (CAT < 10), Gruppe B deutliche Symptome (CAT $\geq 10$ ). Die Gruppen $\mathrm{C}$ und D fassen die Patienten mit häufigen oder schweren Exazerbationen zusammen, jeweils unterteilt in solche mit wenigen (Gruppe C: CAT < 10) oder vielen Symptomen (CAT $\geq 10$ ). Die Messung der Lungenfunktion ist für die Therapiewahl zwar nicht mehr notwendig. Sie behält aber fundamentale Bedeutung in der Diagnostik, und für die Verlaufskontrolle/Prognose.

Der Einsatz von inhalativen Steroiden (ICS) wurde eingeschränkt, so Vogelmeier. Grund war einerseits die Unterlegenheit der ICS/LABA-Kombination gegenüber der dualen Bronchodilatation in der FLAME-Studie. Ein zweiter Grund sei das erhöhte Pneumonieriisiko unter ICS-Therapie. Etwa jeder zweite COPDPatient in Deutschland erhalte ICS, das sei entschieden zuviel, so Vogelmeier.

Eine gut untersuchte Option eines dualen Bronchodilatators mit einfach $\mathrm{zu}$ handhabendem Inhalator sei Aclidinium/Formoterol (Brimica ${ }^{\circledR}$ Genuair $^{\circledR}$ ), berichtete Dr. Volker Töpfer, Ulm. Die zweimal täglich anzuwendende Fixkombination verbessere die Lungenfunktion stärker als die Einzelkomponenten oder eine ICS/LABA-Kombination und verschaffe dem Patienten eine schnelle und über 24 Stunden spürbare Wirkung. V.a. in den Morgenstunden linderte die Therapie die belastenden Symptome wie Hus- ten, Verschleimung und Atemnot. Dadurch waren die Patienten morgens aktiver als unter Placebo oder Aclidinium bzw. Formoterol.

\section{Dr. Dirk Einecke}

- Pressegespräch „2 Jahre Brimica ${ }^{\oplus}$ Genuair ${ }^{\circledast}$ : Die moderne LAMA/LABA-Fixkombination bewährt sich im Praxisalltag"; Berlin, Januar 2017 (Veranstalter: Berlin-Chemie)

\section{Prophylaxe und Therapie von HIT-Thrombosen}

Die heparininduzierte Thrombozytopenie (HIT) ist eine unerwünschte Arzneimittelreaktion, die bei Gabe von unfraktioniertem Heparin und - deutlich seltener - von niedermolekularem Heparin auftreten kann. Bei Patienten mit starkem Verdacht auf HIT oder bestätigter HIT muss die Heparingabe gestoppt und eine Antikoagulation mit einem sofort wirksamen alternativen Antikoagulans begonnen werden, z. B. mit Danaparoid-Natrium (Orgaran ${ }^{\circledast}$ ).

Es besteht aus einer Mischung verschiedener Glykosaminglykane. Vorteilhaft ist laut Aussage von Prof. Andreas Greinacher, Universitätsmedizin Greifswald, dass es sowohl subkutan appliziert, als auch intravenös infundiert werden kann. Aufgrund der langen Halbwertszeit hat es einen stabilen gerinnungshemmenden Effekt. „Danaparoid ist das einzige Antikoagulans, das die durch HIT-Antikörper-vermittelte Zellaktivierung hemmt und damit direkt der Pathogenese der HIT entgegenwirkt", betonte Greinacher.

Dr. Susanne Heinzl

- Symposium "HIT oder nicht HIT? - das ist hier die Frage!", 61. Jahrestagung der Gesellschaft für Thrombose und Hämostaseforschung; Basel, Februar 2017 (Veranstalter: Aspen) 